\title{
Phenomenographic Study of Basic Science Understanding-Senior Medical Students' Conceptions of Fatigue
}

\author{
Niklas Wilhelmsson, Lars Owe Dahlgren ${ }^{1}$, Håkan Hult ${ }^{1}$, Staffan Wirell ${ }^{2}$, Torbjörn Ledin ${ }^{3}$, \\ Anna Josephson ${ }^{4}$ \\ Department of Learning, Centre for Medical Education, Informatics, Management and Ethics, Karolinska Institutet, Stockholm, ${ }^{1}$ Department of \\ Behavioural Sciences and Learning, ${ }^{2}$ Unit of Radiology, Department of Medicine and Care, Faculty of Health Sciences, Linköping University, \\ ${ }^{3}$ Department of Clinical and Experimental Medicine, Faculty of Health Sciences, Linköping University, Sweden and Department of \\ Otorhinolaryngology, County Council of Östergötland, Linköping, ${ }^{4}$ Department of Neuroscience, Karolinska Institutet, Sweden
}

\begin{abstract}
Introduction: Helping students learn to apply their newly learned basic science knowledge to clinical situations is a long-standing challenge for medical educators. This study aims to describe how medical students' knowledge of the basic sciences is construed toward the end of their medical curriculum, focusing on how senior medical students explain the physiology of a given scenario. Methods A group of final-year medical students from two universities was investigated. Interviews were performed and phenomenographic analysis was used to interpret students' understanding of the physiology underlying the onset of fatigue in an individual on an exercise bicycle. Results: Three categories of description depict the qualitatively different ways the students conceptualized fatigue. A first category depicts well integrated physiological and bio-chemical knowledge characterized by equilibrium and causality. The second category contains conceptions of finite amount of substrate and juxtaposition of physiological concepts that are not fully integrated. The third category exhibits a fragmented understanding of disparate sections of knowledge without integration of basic science and clinical knowledge. Discussion: Distinctive conceptions of fatigue based with varying completeness of students' understanding characterized the three identified categories. The students' conceptions of fatigue were based on varying understanding of how organ systems relate and of the thresholds that determine physiological processes. Medical instruction should focus on making governing steps in biological processes clear and providing opportunity for causal explanations of clinical scenarios containing bio-chemical as well as clinical knowledge. This augments earlier findings by adding descriptions in terms of the subject matter studied about how basic science is applied by students in clinical settings.
\end{abstract}

Keywords: Phenomenography, problem-based learning, qualitative methodology, student learning, undergraduate medical education

\section{Introduction}

One of the more long-lived challenges in medical education has been the apparent gap between basic science training and clinical practice, and the associated problem of knowledge transfer for physician-trainees. ${ }^{[1-3]}$ Previous authors have

\begin{tabular}{|l|l|}
\hline \multicolumn{2}{|c|}{ Access this article online } \\
\hline Quick Response Code: & Website: \\
& www.educationforhealth.net \\
\cline { 2 - 3 } & \\
\hline
\end{tabular}

addressed the curricular and educational difficulties of helping students apply their basic science knowledge to clinical situations. ${ }^{[4,5]}$ Focus on more general problem-solving skills, ${ }^{[6]}$ as well as emphasizing medically coherent explanations involving biochemistry, neuroscience, and physiology have been suggested as remedies. ${ }^{[7,8]}$

Physiology, in particular, has been regarded as a field that poses considerable difficulties for students, ${ }^{[9]}$ who develop misconceptions in important areas such as cardiac physiology ${ }^{[10]}$ and respiratory physiology. ${ }^{[11,12]}$ Fyrenius et al. ${ }^{[13]}$ found undergraduate medical students hold conceptions of general principles in physiology characterized by the effects of central and peripheral regulatory mechanisms. Studies

\section{Address for correspondence:}

Dr. Niklas Wilhelmsson, Centre for Medical Education, Department of Learning, Informatics, Management and Ethics, Berzelius väg 3, Karolinska Institutet, 17177 Stockholm, Sweden. E-mail: niklas.wilhelmsson@ki.se 
[Downloaded free from http://www.educationforhealth.net on Wednesday, September 03, 2014, IP: 130.236.236.124] || Click here to download free Android applicat for this journal

Wilhelmsson, et al:: Basic science understanding

find that educational efforts to identify and correct students' misconceptions in the application of physiology principles to clinical situation have had limited success. ${ }^{[14]}$

This study explores the nature of senior medical students' understanding of basic science concepts. In overcoming the perceived gap between basic science and clinical knowledge, integration between preclinical and clinical knowledge areas can be fostered in order to achieve "knowledge-encapsulation". ${ }^{[15]}$ According to this process, biomedical knowledge is encapsulated into condensed concepts of causal models known as clinical knowledge, finally ending up in illness-scripts comprising clinically relevant information about the disease in question. ${ }^{[16]}$ In this way, basic science consisting of detailed causal networks of patho-physiological mechanisms is supposed to be restructured into more comprehensive, high-level concepts. These concepts "encapsulate" the more detailed knowledge. ${ }^{[17,18]}$ With increasing experience, encapsulated concepts form a gradually more important base for clinical decisions. ${ }^{[19]}$ However, while some studies have found a fragile connection between basic science and clinical knowledge, ${ }^{[20,21]}$ others have suggested a critical role for the basic sciences, ${ }^{[22]}$ especially in diagnosing nonroutine cases ${ }^{[2,24]}$ by providing causal links between underlying mechanisms and a patient's clinical manifestation. Recent suggestions have been made to supplement the traditional view of the medical expert, who has been recognized for the amount of knowledge and experience she/he possesses. ${ }^{[25]}$ These attempts at balancing efficiency and innovation in approach to clinical problems in order to account for differences in expert performance in handling medical knowledge.

The aim of the present study is to explore senior medical students' understanding of a basic science scenario by applying basic physiological mechanisms.

\section{Methods}

\section{Context of the study}

The Swedish medical education program comprises 11 semesters (5 1/2 years) during which basic science subject-areas like biochemistry and anatomy are followed by clinical rotations. Students are admitted directly from high school, aged on average around 20 years and the vast majority from a native background. Seven universities offer a medical program after which a 2-year internship leads to license to practice.

Two medical universities, one employing problem-based learning (PBL) (Linköping) and one with a traditional curriculum (Stockholm) were involved in this study. The medical program at the PBL university was divided into three phases: (i) the healthy human (covering basic science topics such as anatomy and physiology), (ii) the ill human (involving disciplines like pathology and pharmacology), and (iii) clinical medicine (comprising clinical rotations). Students studied physiology integrated during the first two years. PBL had been used in this university since 1986, which meant that all teaching-staff were familiar with the basic didactical ideas. Assessment consisted of integrated examinations at the end of each term. At the second university, with a traditional educational program, the curriculum consisted of a preclinical phase occupying the first two years of study with basic science subject-areas, and a clinical phase comprising clinical rotations. Physiology was studied in the beginning of the second year. Lectures and seminars were the most commonly employed teaching-and-learning-activities but there was substantial variation between courses.

\section{Participants}

Twenty medical students participated, ten from each university. All students at both schools were invited during a lecture to take part in a pedagogical test to be included in an educational research project. An equal number of female and male volunteers were selected without information of their prior academic performance. A cinema ticket was given as reward for participation. The interviewed students were all native Swedish, aged between 19 and 26. All students were interviewed at the end of their fifth year, approximately three years after their coursework in physiology.

\section{Data collection}

Semi-structured interviews lasting 25-40 minutes were carried out with a focus on the students' conceptions of physiological fatigue. Interviews were used so students could elaborate in their answers. Interviews covered aspects of biochemistry, physiology, and to some extent neuro-science. The topic as introduced to students was: Imagine a person on an exercise bicycle. The onset of fatigue is often sudden when the workload is increased gradually. Please explain why. Four of the authors performed five interviews each. They participated in a seminar on phenomenographic interview technique directly before the start of the study, and each performed a pilot-interview to ensure confidence in their task. The interviewers were clinical teachers at their respective schools, all with extensive experience in teaching undergraduates and in research in the basic sciences. A senior professor of physiology provided the faculty-interviewers with a correct account of the physiological processes involved in the fatigue scenario posed to the students. Interviewers followed the same interview-manual with follow-up questions to the initial presentation of the problem. They had no information of the student's prior performance on examinations. Upon presentation of the topic, the students were instructed to give a full account of the mechanisms involved in the experience of fatigue. The participants were reminded that the interview was not an oral 
[Downloaded free from http://www.educationforhealth.net on Wednesday, September 03, 2014, IP: 130.236.236.124] || Click here to download free Android applicat for this journal

Wilhelmsson, et al:: Basic science understanding

examination. Probing ${ }^{[26]}$ was applied during the interview to obtain exhaustive descriptions and was performed through (i) repeating (what the student just said), (ii) restoring (to a prior track of discourse), (iii) clarifying-summation (asking for explanation or summarizing one part of the discussion) and (iv) translocation (introducing a new concept not previously mentioned by the student). These procedures were employed to increase transparency of the interview material to reveal any qualitative differences in the students' understanding. The interviews were conducted in the mother-tongue of both interviewer and interviewee, transcribed in verbatim, and then translated into English.

\section{Data Analysis}

A phenomenographic approach ${ }^{[27]}$ was employed in analysis, the aim of which is to discern and characterize the various qualitatively different ways that subjects understand a phenomenon. In this study a lot of attention was also to be paid to how the categories related to each other, that is, in what way the students' understanding differed. It was considered important to capture as many conceptions as possible, to give the study's findings breadth and completeness, since it might be that students from different educational backgrounds may hold different conceptions. There was no attempt to make a comparison between the conceptions held by the students of the two schools.

The written transcripts of each interview were analyzed individually by all authors, after which they met and discussed the findings in detail. The completeness of each interview was assessed depending on the included number of sub-areas (Krebs's cycle, type of metabolism, muscle contraction, regulation of both respiration and heart activity) and their respective accuracy, as well as the degree of developed relations between them. Aspects considered in this sense included depth (richness of detail), breadth (number of organ-systems covered), and stringency (speed and structure of the answer).

In the analysis of the interview transcripts, the students were seen as bearers of different conceptions regarding physiological fatigue. All the statements containing conceptions were initially analyzed across individuals and referred to a common pool of meanings. Following this, each individual interview was treated as a whole and assigned to a category.

We set out to describe qualitative differences among students' various understandings of fatigue. Having closely penetrated the transcripts, they were subjected to comparison with respect to similarities and differences and grouped according to these features. Key concepts, serving as markers for different qualitative aspects of the students' reasoning, were identified. In an iterative process the categories presented in the 'Results' section were constructed through formulation of their respective core-content, reorganization of the category-system, clarification of the dividing boundaries to make their essence distinct, and labeling them accordingly. ${ }^{[28]}$ Thus, the categories represent an exhaustive depiction of the critical variation in the ways of understanding the phenomenon. Participants' quotations presented in the 'Results' section illustrate typical conceptions of that particular category, to demonstrate the core of the category and delimit it from others.

\section{Results}

\section{The analysis yielded three categories of description}

Category A: Chain of mutually interdependent physiological and biochemical sub-mechanisms

In this category the relation between sensors and effectors is central. Cause-effect relations are clearly explained and used to interpret both the sensory aspect (information to the brain) and the effectuate aspect (resulting modulations governed by the brain) of the situation, for example, the activation of accessory breathing muscles as a response to a detected high level of $\mathrm{CO}_{2}$ and lactate in glomus caroticus. This two-way causality (sensory causality and effectuate response) provides answers found within this category with an alignment, making clear the role of each physiological mechanism at any given point in the course of events. Relations between the systems involved are clear-cut, as are which systems are inhibiting and exciting in a certain process. Students' answers within this category typically contain all relevant organ-systems, and provide an in-depth description of bio-chemical interactions to the resulting cerebral adjustments as mediated by intermediary systems.

"And that brings on acidosis in the blood stream, which has the result that you try to ventilate it out in the form of carbon dioxide.... And there is no time for the lactate to be converted in this long-running work-load back to new glucose in the liver, in this gluconeogenesis, so there will be a accumulation of lactate in the blood-stream which brings on pain, hyperventilation etc., Then we have the heart rate, which rises. That is also a response to the tissue's demand of new oxygen and energy. The hyperventilation... is caused by several things, but you have chemo-receptors in, I think, the main vessels, or at least the brain has chemo-receptors that detect the level of carbon dioxide and send signals to the respiratory centre to increase breathing-rate and heart-rate."

A hallmark of this category is the notion of equilibrium. The breadth of the line of reasoning allows for relating and comparing parameters that have been brought up. Building on the relative impact these parameters have on the situation, their internal connection establishes an understanding of the balance, which is about to derange.

"Right, lactate is a product from the glycolysis... that forms under anaerobic conditions and it's not on or off, it's not like you either go anaerobic or aerobic, but as I mentioned earlier that (lactate) is also produced during aerobic combustion, but in lesser quantities." 
[Downloaded free from http://www.educationforhealth.net on Wednesday, September 03, 2014, IP: 130.236.236.124] || Click here to download free Android applicat for this journal

Wilhelmsson, et al:: Basic science understanding

Closely connected to the concept of equilibrium is the concept of threshold. When the equilibrium is displaced by a certain factor, this factor must, in turn, have been affected by yet another. The threshold crossed resulting in disequilibrium becomes central to the explanation. The following quote gives two examples of situations were thresholds play an important role in relation to the situation.

"Now, we've been speaking about cardiac output and heart rate, and that they rise together, but there is a threshold where they don't rise that much any longer, the pulse goes through the roof but cardiac output remains constant unfortunately..."

In this category, the way of conceptualizing the situation includes making use of a broad range of physiological facts and a focus on the cause of a displaced equilibrium. These facts are organized in a comprehensive way making the links between different courses of events easily approachable through their causal nature. The resulting alignment in the explanation is therefore characterized by a precision in definition since the aim of each sub-system is to provide a reason for the next system. Concentrating the reasoning around the governing principles of a certain event does not, however, mean that there is a loss of detail. Indeed, the statements found in this category prove that the opposite is true: Focusing on the whole goes hand in hand with recall of factual and terminological knowledge.

Category B:Juxtaposed physiological and biochemical mechanisms This second category bears some resemblance to the first category in that it contains causal relations. However, these are not of a reciprocal kind and general causality does not feature as a main characteristic of the category. Here, the accurate reproduction of the included bio-chemical mechanisms and organ-systems are the focus. All of the involved systems are generally discerned and most of them elaborated upon adequately, but their internal relations are not accounted for in a thorough way. Instead, explanations of the connections between the various systems are juxtaposed in students' comments rather than casually linked. In this way of understanding the scenario, a horizontal line-up of all possible levels of physiological processes related to the experience of fatigue is emphasized. Compared to the previous category, where a more vertical disposition was seen, a certain sense of consequence in alignment is lost. Furthermore, the relevance of a particular process for the greater chain of events becomes obscure to the student, since it is not connected to the whole in a consistent way. Inappropriately used medical terms without adequate precision also mirrors this preoccupation with juxtaposed processes at the expense of coherent causal linking. The precision in the use of terms becomes overshadowed by the completeness of factors involved. However, no major misunderstandings are found among answers in this category.
Some students juxtaposed organ-systems to explain why anaerobic metabolism arises. Emphasis was given to identifying an abundance of factors involved, not on the governing and regulatory steps that give rise to the situation. "Er, I think it is saturation of oxygen, or supply of oxygen rather. Oxygen, the amount of oxygen that can be taken up and transported, in haemoglobin that is, and then there is the muscle, yes OK, the heart's capacity to pump blood, for example, and muscle mass in addition to the amount of easily accessible energy. And, yes, the storage of glycogen and glucose in the liver and..."

A second feature of this category is the conception of a finite amount of substrate. If in the prior category the situation was perceived as one of a displaced equilibrium, with two or several systems running parallel until a threshold stage was reached, this category contains statements concerning the limited supply of combustible substrate. Once the compensatory mechanisms have been exhausted the fuel tank has gone empty, and this gives rise to the modified combustion:

"Well, I think of it like this, that when the vessels are widened to a maximum and the heart's pounding and when you... it's about mechanisms of compensation being pushed to their limits, then there's like, nothing more to do, you simply have to stop cycling."

The horizontal way of organizing the answer sometimes results in an unclear relevance of the aspects raised. There is a fragmentation to the alignment of the argument. Although accurate, the following quote is imprecise and the relevance less obvious.

"Carbon dioxide. what is that intermediate step called now?. the hydrogen ions are being buffered, I don't remember the intermediate step there, but the hydrogen ions come out of the water vapour, carbon dioxide. I think I can picture it in front of me, but not explain it exactly, but there is a formula where one can see that if carbon dioxide disappears then the hydrogen ions are buffered and one adjusts to that quickly. Then you can also adjust to it with a buffer, with the help of the kidneys, but the buffer is instantaneous and the kidneys take longer. Oh, I'm lousy at explaining this, it's fascinating how much you forget!"

Category C: Fragmented physiological and biochemical mechanisms

The central feature of the third category is fragmentation. Mostly, the answers found here are insufficient as an account of the presented situation. They are characterized by a limited use of physiological concepts and causal relations, as well brevity in explanation. When memory about bio-chemical knowledge fails them, students focus on clinical medicine. In order to keep the discussion close to the original question demands a great deal of steering by the interviewer. As opposed to the prior category, students found here are not able to make use 
[Downloaded free from http://www.educationforhealth.net on Wednesday, September 03, 2014, IP: 130.236.236.124] || Click here to download free Android applica for this journal

Wilhelmsson, et al:: Basic science understanding

of the steering provided by the interviewer to support their reasoning. Misconceptions are common, although they do not qualify as a central element of the category since the students prefer to stop explaining than provide an incorrect answer.

The following reflects the fragmentation heard within this category, in its inexact report on fatigue. Definitions are absent, the language employed is vague and irrelevant systems are mentioned.

"Right, I don't remember what it is exactly when you exercise, when you are out of oxygen and start to work anaerobic... above all there is a large production of lactate, like, and that is a strong signal that now it's time to quit. ATP is involved here somehow, and some other stuff that I don't remember the name of, which are also stop-signals in a way, but also shouts to the blood, 'Hey, come here, I need some more energy over here!' and you can have redistribution of the blood to where it's needed."

The careless handling of medical terms leads to imprecision bordering misconception. There is also an echo of the finite amount of fuel also seen in category B explanations. The resulting characteristic dead-end illustrates the student's unresponsiveness to steering by the interviewer.

"It takes longer and costs more energy to go on like this... and this thing about working out or not, and the blood-supply, you can only do so much of this anaerobic metabolism, and that is why... you hit the wall. You have to compensate all the time by breathing and the blood goes round and the heart pumps more blood, more oxygen to the blood and more carbon dioxide to the lungs and out, and so on, so you get rid of this... And when it's forced to stop, like, that has to be when you simply can't compensate any longer...no, I can't say exactly how it works. Signals up to the brain...no, I don't know."

The subject in the following quote concludes that the answer is not of great importance, presumably meaning for a practicing doctor.

"That should be more like where the signals go, from here to there, generally I know that, but maybe not how this comes about in the system. I know that if you do this, then that will result, but how it works...Although, I didn't check up on this much either, can't say it feels very important, I don't feel like I have to know this, but I can check it up if I want to... it doesn't feel very important."

The number of students found in each category was A ( 5 students), B (7 students) and C (8 students).

\section{The relation between the categories}

The outcome-space constituted by the categories is hierarchically ordered, which means that all conceptions found in the $C$ category are also present in categories A and B. And while both $B$ and $C$ lack some of the conceptions displayed in the A category, C displays even less than B. In order to depict this hierarchical relationship, an example of reasoning typical of each category is listed in Table 1.

The shift from aerobic to anaerobic metabolism was held central in the explanations among all participating subjects. However, this shift is outlined quite differently in the three categories. The importance of anaerobe metabolism for the experience of fatigue is only briefly explained in a category $C$ answer, while an answer in the B category clarifies that the shift is due to a decreased supply of oxygen in the muscle cell. In a category A answer, the detailed account of each process is less emphasized to give room for causal relations, placing the single muscle-cell in a meaningful context and making its role understandable in relation to this context.

\section{Discussion}

The qualitative data presented in this investigation is obtained from a second-order perspective. This means that physiological fatigue as conceptualized by the participants is the object of research, not the phenomenon of physiological fatigue itself. This type of analysis offers the possibility of descriptions of a complex issue in its full richness. The categories of description depict three qualitatively different ways of understanding physiological fatigue. Instead of measuring the amount of knowledge remembered, our categories aim at describing the nature of that knowledge in terms of its own content. ${ }^{[29]}$ This makes it possible to characterize the conceptions held by the students as subject-matter specific, self-contained data about the experience of the phenomenon of interest without reference to preconceived patterns of thought generated from either previous research results or theory. The empirical voice is given priority over theoretical constructs.

Table 1: Example of statements depicting the hierarchical relation among the categories

Category No Quote

C C4 "It has to do with... when a sugar-molecule can't become...that is, it can't travel all the way through this chain of events that takes place in the cell...it will enter, I mean, it will create lactic acid, lactate..."

B

B4 "Yes, that is because there is a storage of energy in the muscle cells and when you start exercising you make use of that stored energy first, aerobic metabolism, and then when the stored energy is starting to be used up and the oxygen supply starts to decrease, then you go over to anaerobic energy, and then lactate is produced, you get pain in your muscles and you stop the exercise"

A

A4 "Numbness, yes, you'll have that, even though there are good buffering systems and the like, you'll definitely end up with a lowered $\mathrm{pH}$ locally in the muscle, and also easily detected in the blood stream, but above all in the muscle, and this will give rise to vasodilatation to increase blood flow in the area, but also an exit of fluid to the interstitium, and so on, a subjectively painful, swollen muscle, it's a kind of local acidosis, an exit of fluid. There are several fairly vaso-active metabolites created in anaerobic metabolism" 
[Downloaded free from http://www.educationforhealth.net on Wednesday, September 03, 2014, IP: 130.236.236.124] || Click here to download free Android applicat for this journal

Wilhelmsson, et al:: Basic science understanding

Identifying what a learner understands the task at hand to be about is essential in any learning environment, ${ }^{[30]}$ as this will inevitably influence the outcome of learning. ${ }^{[31]}$ Consequently, the categories can be used in making explicit how the situation was understood by the students, since they reflect the perceptions that governed the students' approach. A pragmatic instructional strategy can then be adapted to reinforce certain critical aspects of the subject-matter (e.g., equilibrium and causality as seen in category A), as a deficit in causal linkage between areas seems to carry a risk of compartmentalization of the knowledge (as seen in category C). A more extensive use of detailed basic physiological knowledge was used among students with category A responses than with category B responses, and even less with category $C$ responses. Therefore, it would seem that students exhibiting a more thorough understanding would also perform better in examinations and assessments, although no such data was available. Emphasizing general principles has been previously suggested in order to promote students' understanding of physiology. ${ }^{[32,33]}$ It is assumed that students, in realizing similarities on a conceptual level between different physiological situations, will be able to transfer their problem solving ability between areas of application and through that gain a better understanding. These findings, arrived at by analyzing the subject area of physiology, appear to correspond to certain features of the category system; causally linked events conducted by governing principles. They have also been observed in a phenomenographic inquiry into students' conceptions of blood pressure regulation. ${ }^{[13]}$ The students in our study were not asked about the transferability of the principles involved, but there is an association between the use of causal principles and a more complete understanding, as demonstrated in category $\mathrm{A}$.

Work by Woods and colleagues ${ }^{[22]}$ similarly suggests that the fundamental function of basic science in medical education is to create links between patho-physiological mechanisms and clinical situations. Although the situation presented to students in this study is not necessarily clinical, it is related to clinical practice through application of basic physiological mechanisms. We chose this scenario as it would maximize the basic science content in the participants' explanations, and because an understanding of pathological processes and diagnostics requires an understanding of normal function physiology. Here, we have attempted to address students' level of basic science knowledge three years after they completed their physiology coursework and have not limited the scope to diagnostic skills. There seems to be an association within the students between an accurate use of basic science models of explanation and a more detailed memory or knowledge of basic science, as seen in category A responses. ${ }^{[34]}$

Medical students' understanding of physiological phenomena in a preclinical setting has previously been investigated by
Fyrenius et al. ${ }^{[13]}$ In their study, four categories of description depict increasingly complex ways of understanding the underlying principles in human physiology. Causal reasoning featured as an overall model of explaining physiological processes, while conditions for transferability of a certain principle to other areas of application represented more advanced forms of understanding. Also in our study, causal reasoning plays an important role in linking basic science with clinical context. Students exhibiting a better understanding (category A) made more use of causal reasoning, as alignment between sub-processes was a central aspect of their explanation. However, within a clinical context other studies suggest that problem-specific knowledge is associated with diagnostic skill to a higher degree than generic knowledge. ${ }^{[35]}$

In recall of clinical information, experts and novices differ in their ability to separate relevant information from irrelevant. ${ }^{[3]}$ Although our study did not include experts, but rather only advanced students, relevance of the explanation given still featured as a central concept in the outcome-space: A category A explanation consists of clear facts ordered according to relevance, while juxtaposing biochemical mechanisms (category $\mathrm{B}$ ) and fragmenting the knowledge (category C) necessarily blurs the relevance of each mechanism. In the present study, all participating students managed to mention the shift from aerobic to anaerobic metabolism. The degree of elaborated inter- and intra-related connections between the included organ systems delimits the categories from each other and determines the differences in how the knowledge is organized.

The scope in the present study was broad: How do senior medical students understand a basic physiological problem? The analysis was focused on the qualitatively different ways of understanding the participants provided. It was not performed with regards to assumed differences between the two cohorts, instead, the interviews were all treated alike and the conceptions they presented were categorized as described in the 'Results' section. Some limitations regarding the generalizability of the findings follow from the design of the study. The strategic sampling procedure that was used does not allow for general comparisons between the two modes of instruction, and even less the two educational theories that underlie them. Nor can it be assumed that other last-year medical students would hold exactly the same conceptions of fatigue as the students in the present study. However, it is likely that the analysis has captured essential aspects of students' understanding of physiology in a clinical setting that are transferable to other contexts and clinical situations.

Trustworthiness of the present study should be assessed by its inclusion of participants, choice of methodology and 
[Downloaded free from http://www.educationforhealth.net on Wednesday, September 03, 2014, IP: 130.236.236.124] || Click here to download free Android applica for this journal

analysis, and the degree to which the results correspond to the participants' provided experience. ${ }^{[37]}$ The group of participants was composed to secure breadth of experience regarding the phenomenon. The open-ended interview allowed for rich and robust descriptions and the provided answers were continuously corroborated with the interviewee. The phenomenographic analysis delivered useful results informing teaching practice within the subject-area.

In addition to providing a theoretical form of methodologyanchored concepts, the categories of students' understanding identified in this study can be used in educational practice as guidance to avoid threatening misconceptions. According to earlier research, the view students' hold of the subject-area influences their approach to learning as well as the outcome of learning. ${ }^{[31]}$ If the categories are viewed as students' different attempts toward understanding, the categories can help adapt teaching methods to reinforce certain aspects of physiology (such as equilibrium) and reveal misconceptions.

Our study shows that phenomenographic analysis can be used to identify educationally important differences in the understanding of physiology and provide tools to inform teaching practice. Moreover, qualitative approaches can help to form new ways of dealing with issues researched in medical education, even those raised within other traditions. Without losing their respective methodological peculiarities, research communities should be able to appreciate the strength of different approaches in working toward a shared understanding of the areas we research. Within this particular research field, cause and effect relations have been extensively studied, but the present study closely examines how most students succeeded in not getting lost in the wood, despite some difficulties in seeing the wood for all the trees.

\section{Acknowledgements}

This research was funded by grant no. SLL 20060812 from the Fund for pedagogical research in health care, Stockholm City Council.

\section{References}

1. Flexner A. Medical Education in the United States and Canada: A report to the Carnegie Foundation for the advancement of teaching. Bulletin no. 4. New York, Updike, 1910.

2. Cole L. What is wrong with the medical curriculum? Lancet 1932;110:253-4.

3. Prince K, Van De Wiel M, Scherpbier AJ, Can Der Vleuten CP, Boshuizen HP. A qualitative analysis of the transition from theory to practice in undergraduate training in a PBL-medical school. Adv Health Sci Educ 2000;5:105-16.

4. Albanese MA, Mitchell S. Problem-based Learning. A review of literature on its outcomes and implementations. Acad Med 1992;68:52-81.

5. Norman GR, Can der Vleuten CP, Newble DI. International Handbook of Research in Medical Education. Boston: Kluwer; 2002.
6. Norman GR, Schmidt H. The psychological basis of problem-based learning: A review of the evidence. Acad Med 1992;67:557-65.

7. Patel VL, Groen GJ, Norman GR. Reasoning and Instruction in Medical Curricula. Cogn Instr 1993;10:335-87.

8. Hmelo C, Gotterer G, Bransford J. A theory-driven approach to assessing the cognitive effects of PBL. Instr Sci 1997;25:387-408.

9. Modell HI, Micheal JA, Adamson T, Goldberg J, Horwitz BA, Bruce DS, et al. Helping undergraduates repair faulty mental models in the student laboratory. Adv Physiol Educ 2000;23:82-90.

10. Michael JA, Wenderoth MP, Modell HI, Cliff W, Horwitz B, McHale $\mathrm{P}$, et al. Undergraduates' understanding of cardiovascular phenomena. Adv Physiol Educ 2002;26:72-84.

11. Michael JA. Students' misconceptions about perceived physiological responses. Adv Physiol Educ 1998;19:90-8.

12. Michael JA, Richardson D, Rovick A, Modell H, Bruce D, Horwitz B, et al. Undergraduate students' misconceptions about respiratory physiology. Adv Physiol Educ 1999;22:127-35.

13. Fyrenius A, Silén C, Wirell S. Students' conceptions of underlying principles in medical physiology: An interview study of medical students' understanding in a PBL curriculum. Adv Physiol Educ 2007;31:364-9.

14. Morton JP, Doran DA, MacLaren DP. Common student misconceptions in exercise physiology and biochemistry. Adv Physiol Educ 2008;32:142-6.

15. Schmidt HG, Boshuizen HP. On acquiring expertise in medicine. Educ Psych Rev 1993;5:1-17.

16. Schmidt HG, Norman GR, Boshuizen HP. Acad Med 1990;10:611-21.

17. Rikers RM, Schmidt HG, Moulaert V. Biomedical Knowledge: Encapsulated or two worlds apart? App Cogn Psych 2005;19:223-31.

18. De Bruin A, Schmidt H, Rikers R. The role of basic science knowledge and clinical knowledge in diagnostic reasoning: A structural equation modelling approach. Acad Med 2005;80:765-73.

19. Rikers R, Loyens S, Schmidt H. The role of encapsulated knowledge in clinical case representations of medical students and family doctors. Med Educ 2004;38:1035-43.

20. Patel VL, Groen GJ, Scott HM. Biomedical knowledge in explanations of clinical problems by medical students. Med Educ 1988;22:398-406.

21. Norman GR, Brooks LR. The non-analytical basis of clinical reasoning. Adv Health Sci Educ Theory Pract 1997;2:173-84.

22. Woods N, Brooks L, Norman G. The role of basic science in clinical diagnosis: Creating coherence among signs and symptoms. Med Educ 2005;39:107-12.

23. Woods N, Brooks L, Norman G. The role of biomedical knowledge in diagnosis of difficult clinical cases. Adv Health Sci Educ 2007a; 12:417-26

24. Woods N, Brooks L, Norman G. It all makes sense: Biomedical knowledge, causal connections and memory in the novice diagnostician. Adv Health Sci Educ 2007b; 12:407-15.

25. Mylopoulos M, Woods NN. Having our cake and eating it too: Seeking the best of both worlds in expertise. Med Educ 2009;43:406-13.

26. Abrandt M. Learning Physiotherapy: The impact of formal education and professional experience, Dissertation, Linköping studies in education and psychology no 50, Department of Education and Psychology, Linköping University, Linköping, 1997.

27. Marton F. Phenomenography-describing conceptions of the world around us. Instr Sci 1981;10:177-200.

28. Wilhelmsson N, Dahlgren LO, Hult H, Scheja M, Lonka K, Josephson A. The Anatomy of Learning Anatomy. Adv Health Sci Educ 2009;??

29. Dahlgren LO. Outcomes of learning. In: The Experience of Learning ( $2^{\text {nd }}$ ed.), Marton F, Hounsell D, Entwistle N, editors. 
[Downloaded free from http://www.educationforhealth.net on Wednesday, September 03, 2014, IP: 130.236.236.124] || Click here to download free Android applicat for this journal

Edinburgh: Scottish Academic; 1997. p. 23-38.

30. Wilhelmsson N. From basic science knowledge to clinical understanding, Dissertation, Department of Learning, Informatics, Ethics and Management, Karolinska Institutet, Stockholm, 2010.

31. Calman KC. Medical Education, Past, Present and Future, Handing on learning. Philadephia: Elsevier; 2007.

32. Michael J, Modell H, McFarland J, Cliff W. The "core principles" of physiology: What should students understand? Adv Physiol Educ 2009;33:10-6.

33. Modell H. How to help students understand physiology? Emphazise general models. Adv Physiol Educ 2000;23:101-7.

34. Dahlgren LO, Pålsson E. What is left? Paper presented at The sixth International SRHE Conference on Higher Education. Lancaster, Great Britain, 1984.
35. Coderre S, Jenkins D, Mclaughlin K. Qualitative differences in knowledge structure are associated with diagnostic performance in medical students. Adv Health Sci Educ 2009;14:677-84.

36. Patel VL, Groen GJ, Frederiksen CH. Differences between medical students and doctors in memory for clinical cases. Med Educ 1986;20:3-9.

37. Giacomini MK, Cook DJ. Users' Guide to the medical litterature: XXIII qualitative research in health care. A. Are the results of the study valid? J Am Med Assoc 2008;284:357-62.

How to cite this article: Wilhelmsson N, Dahlgren LO, Hult H, Wirell S, Ledin T, Josephson A. Phenomenographic study of basic science understandingsenior medical students' conceptions of fatigue. Educ Health 2013;26:156-63.

Source of Support: Nil. Conflict of Interest: No. 